Check for updates

Cite this: RSC Adv., 2017, 7, 27506

Received 23rd February 2017

Accepted 7th May 2017

DOI: $10.1039 / \mathrm{c} 7 \mathrm{ra02280h}$

rsc.li/rsc-advances

\section{Novel protonated Fe-containing mesoporous silica nanoparticle catalyst: excellent performance cyclohexane oxidation $\uparrow$}

\begin{abstract}
Mohammad Reza Sazegar, (iD * Aysan Dadvand and Ali Mahmoudi
A mesoporous silica structure (MSN) was synthesized using the sol-gel method, followed by iron incorporation and protonation which afforded Fe-MSN and H/Fe-MSN with Si/Fe ratios of 20. Nitrogen physisorption confirmed their mesoporous structures with pore diameters of $3.9 \mathrm{~nm}$. Iron incorporation decreased the degree of catalyst crystallinity. Fe-MSN and H/Fe-MSN catalyzed the oxidation of cyclohexane to cyclohexanone using hydrogen peroxide at $298 \mathrm{~K}$ in $1 \mathrm{~h}$. The significant advantages of these catalysts were high conversion, short reaction time, easy work-up, and compatibility with various organic and aqueous solvents. The cyclohexanone product was obtained with excellent conversions of 86 and $97 \%$ at $298 \mathrm{~K}$ using Fe-MSN and H/Fe-MSN, respectively. H/Fe-MSN catalyst gave a higher conversion than Fe-MSN. A comparative study of cyclohexane oxidation in the presence of $\mathrm{H}_{2} \mathrm{O}_{2}$ over $\mathrm{H} / \mathrm{Fe}-\mathrm{MSN}$ heterogeneous catalyst showed that $\mathrm{H} / \mathrm{Fe}-\mathrm{MSN}$ had excellent activity at low temperature.
\end{abstract}

\section{Introduction}

Mesoporous silica nanoparticles (MSN) have been widely used in various scientific fields, including medicinal chemistry, environmental science, and petrochemical industries, and in chemical reactions for their special properties, such as large surface area, and high and homogenous pore size and volume..$^{1-3}$ Although, pure mesoporous silica nanomaterials have limited catalytic activities, metal-loaded MSN have been reported as active catalysts. ${ }^{4,5}$ Recently, the incorporation of $\mathrm{Al}^{2}$, $\mathrm{Ti}^{6}, \mathrm{Ni}^{4}$, and $\mathrm{Mn}^{7}$ into MCM-41 was reported to catalyze various cracking, methanation, and oxidation reactions. Mesoporous silica materials with magnetic properties have been prepared using $\mathrm{Fe}, \mathrm{Co}, \mathrm{Ba}$, and $\mathrm{Ni}^{8-10}$ and applied as magnetic adsorbents to remove organic and inorganic pollutants.

Modified mesoporous silica nanomaterials are capable of catalyzing the oxidation of organic compounds. Fe-containing MCM-41 (denoted as Fe-MCM-41) has shown great potential as a catalyst in many reactions and as a sensor for molecular identification. ${ }^{8}$ Fe-MCM-41 has shown good catalytic activity in oxidation reactions. ${ }^{11,12}$ Continuing efforts are being made to improve or synthesize new catalysts to oxidize cyclohexane under mild conditions. ${ }^{12,13}$ Ti-MCM-41 has been used to catalyze the epoxidation of cyclohexene with $\mathrm{H}_{2} \mathrm{O}_{2},{ }^{6}$ while Mn-MCM-41 has been prepared and shown to have high selectivity for stilbene epoxidation using tert-butyl peroxide or TBHP. ${ }^{7}$

Dept. of Chemistry, Fac. of Science, North Tehran Branch, Islamic Azad University, Tehran, Iran.E-mail: m_r_sazegar@yahoo.com; Tel: +98-2122262564

$\dagger$ Electronic supplementary information (ESI) available. See DOI: $10.1039 / \mathrm{c} 7 \mathrm{ra} 02280 \mathrm{~h}$
In this study, Fe-grafted MSN (denoted Fe-MSN) and protonated iron-containing MSN (denoted H/Fe-MSN) were synthesized using the sol-gel method, and their activities and magnetic properties were investigated in cyclohexane oxidation. Reaction conditions for cyclohexane oxidation were optimized for a high oxidation process. The physical properties of the catalysts were confirmed by nitrogen physisorption, XRD, FTIR, XRF, ICP, XPS, and VSM. The products were confirmed by ${ }^{1} \mathrm{H}$ and ${ }^{13} \mathrm{C}$ NMR, and melting point measurements.

\section{Experimental}

\section{Synthesis of Fe-MSN catalyst}

Fe-MSN and H/Fe-MSN were synthesized from MSN using postsynthesis methods, followed by protonation for H/Fe-MSN. Cetyltrimethylammonium bromide (СТAB, $7.44 \mathrm{~g}$ ) was dissolved in a solution containing water $(170 \mathrm{~mL})$, ethanol $(40 \mathrm{~mL})$, and aqueous sodium hydroxide solution $(20 \mathrm{~mL}, 10 \%)$. After stirring vigorously for approximately $30 \mathrm{~min}$ at $298 \mathrm{~K}$, tetraethyl orthosilicate (TEOS, $17.68 \mathrm{~mL}$ ) was added to the mixture. The resulting mixture was stirred for an additional $2 \mathrm{~h}$ at $298 \mathrm{~K}$ and allowed to rest for $20 \mathrm{~h}$ at the same temperature. The sample was collected by centrifugation at $20000 \mathrm{rpm}$ for $30 \mathrm{~min}$ and washed with deionized water and absolute ethanol three times. Surfactant was removed by washing the MSN $(1 \mathrm{~g})$ with a solution of $\mathrm{NH}_{4} \mathrm{NO}_{3}(0.3 \mathrm{~g})$ in ethanol $(40 \mathrm{~mL})$ at $298 \mathrm{~K}$. The surfactant-free product was collected by centrifugation, dried at $383 \mathrm{~K}$ for $20 \mathrm{~h}$, and calcined in air at $823 \mathrm{~K}$ for $3 \mathrm{~h}$. The sample was prepared by grafting iron onto the pure MSN, followed by centrifugation, drying at $383 \mathrm{~K}$ for $20 \mathrm{~h}$, and calcination in air at 
$823 \mathrm{~K}$ for $3 \mathrm{~h}$. Ferric nitrate $\left(\mathrm{Fe}\left(\mathrm{NO}_{3}\right)_{3} \cdot 9 \mathrm{H}_{2} \mathrm{O}\right)$ was used as an iron precursor. Iron loading occurred via an impregnation method ( $\mathrm{Si} / \mathrm{Fe}$ molar ratio, $20: 1$ ). Fe-grafted MSN was denoted as FeMSN.

\section{Synthesis of $\mathbf{H} / \mathrm{Fe}-\mathrm{MSN}$ catalyst}

Protonated Fe-MSN (H/Fe-MSN) was prepared by shaking Fe-MSN $(1 \mathrm{~g})$ in an aqueous solution of $\mathrm{NH}_{4} \mathrm{Cl}(2.5 \mathrm{~g}$ in 50 $\mathrm{mL}$ water) at $298 \mathrm{~K}$ for $16 \mathrm{~h}$, followed by removal of the solution, drying at $383 \mathrm{~K}$ for $20 \mathrm{~h}$, and calcination at $823 \mathrm{~K}$ for $3 \mathrm{~h}$ in air.

\section{Catalyst characterization}

Catalyst crystallinity was measured using a Bruker Advance D8 $\mathrm{X}$-ray powder diffractometer with $\mathrm{Cu} \mathrm{K} \alpha$ radiation $(\lambda=1.5418 \AA$ ) as the diffracted monochromatic beam, operating at $40 \mathrm{kV}$ and $40 \mathrm{~mA}$. The bulk $\mathrm{Si} / \mathrm{Fe}$ ratios of the catalysts were determined using X-ray fluorescence spectroscopy (XRF, Bruker S4 Explorer) using $\mathrm{Rh}$ as the anode target material and operated at $20 \mathrm{~mA}$ and $50 \mathrm{kV}{ }^{14}$

Nitrogen physisorption analysis was conducted on a Quantachrome Autosorb-1 at $77 \mathrm{~K}$. Before measurement, the sample was evacuated at $573 \mathrm{~K}$ for $3 \mathrm{~h}$. Scanning electron microscopy with energy dispersion X-ray spectrometry (SEM-EDX) was conducted on a JEOL JSM-6701 $\mathrm{F}$ to observe the morphology and perform elemental analysis. Before observation by SEM, the sample was coated with Pt using a sputtering instrument. The morphology and average particle size of the catalyst were estimated from transmission electron microscopy (TEM) images captured using a JEOL JEM-2100 transmission electron microscope. Prior to TEM measurements, the powder samples were ground and subjected to ultrasonic treatment in hexane for $10 \mathrm{~min}$. A drop of the suspension was dried on a copper TEM sample grid. ${ }^{3}$

Fourier transform infrared (FTIR) measurements were carried out using an Agilent Carry 640 FTIR Spectrometer. The catalyst was prepared as a self-supported wafer and activated under vacuum at $673 \mathrm{~K}$ for $3 \mathrm{~h} .{ }^{15} \mathrm{UV}$-vis spectra were obtained on a Thermo Spectronic spectrophotometer model Genesys 10UV, from 300-700 nm using a $1 \mathrm{~cm}$ quartz cuvette.

The amount of iron in the samples was identified using inductively coupled plasma with optical emission spectrometry (ICP-OES, Varian 735). The samples were digested using a traditional acid method ( $\mathrm{HCl}, \mathrm{HF}, \mathrm{HClO}_{4}$, and $\left.\mathrm{HNO}_{3}\right)$, diluted adequately, and analyzed for Fe.

The magnetic properties of the catalysts were measured using a vibrating sample magnetometer (VSM, LakeShore 7037/ 9509-P). ${ }^{16}$ X-ray photoelectron spectroscopy (XPS) was performed using an ESCALAB 250Xi spectrometer armed with an $\mathrm{Al}$ K $\alpha$ X-ray source (Thermo Fisher Company). ${ }^{17}$ The scans were conducted and data plotted with respect to the binding energy.

Melting points were taken on a Kofler hot stage apparatus and are uncorrected. ${ }^{1} \mathrm{H}$ and ${ }^{13} \mathrm{C}$ NMR spectra were recorded on a Bruker FT-500 spectrometer, using TMS as the internal standard.

\section{Catalytic activity}

The catalytic activity of the H/Fe-MSN catalyst was investigated in the cyclohexane oxidation reaction. Details of this study are provided in the following section.

\section{Typical experimental protocol}

To a stirring mixture of cyclohexane $(0.02 \mathrm{~mol})$ was added hydrogen peroxide $(0.04 \mathrm{~mol})$ and $\mathrm{H} / \mathrm{Fe}-\mathrm{MSN}(0.01 \mathrm{wt} \%)$ at room temperature. Stirring was continued for $30 \mathrm{~min}$ and the mixture was heated at $323 \mathrm{~K}$ under an air atmosphere for $3 \mathrm{~h}$. The sample was taken at regular intervals and the concentrations of cyclohexane and cyclohexanone were identified by gas chromatography-mass spectrometry (GCMS, HP 5971 detector) and analyzed using a chrompak CP 9000 GC equipped with an RTX-50 capillary column and the same ionization detector. Dodecane was chosen as the internal standard.

The conversion of cyclohexane to cyclohexanone $\left(X_{\text {oxidation }} \%\right)$, yield of cyclohexanone $\left(Y_{\text {oxidation }} \%\right)$, and selectivity for cyclohexanone $\left(S_{\text {oxidation }} \%\right)$ were calculated according to eqn (1)-(3), respectively:

$$
\begin{gathered}
X_{\text {oxidation }}(\%)=\frac{[\mathrm{KET}]_{\mathrm{i}}-[\mathrm{KET}]_{\mathrm{f}}}{[\mathrm{KET}]_{\mathrm{f}}} \times 100 \\
Y_{\text {oxidation }}(\%)=\frac{n_{\mathrm{y}}}{n_{\mathrm{c}}} \times 100 \\
S_{\text {oxidation }}(\%)=\frac{n_{\mathrm{y}}}{n_{\mathrm{i}}-n_{\mathrm{f}}} \times 100
\end{gathered}
$$

where $[\mathrm{KET}]_{\mathrm{i}}$ and $[\mathrm{KET}]_{\mathrm{f}}$ are the initial and final cyclohexane concentrations, $n_{\mathrm{y}}$ and $n_{\mathrm{c}}$ are the yielded and calculated moles of cyclohexanone, and $n_{\mathrm{i}}$ and $n_{\mathrm{f}}$ are the initial and final moles of cyclohexane, respectively.

\section{Reusability testing}

A catalyst reusability test was carried out for H/Fe-MSN in cyclohexanone production (\%) five times at $298 \mathrm{K.}{ }^{18}$ The reactivity was determined by washing with hot dichloromethane (2 $\times 10 \mathrm{~mL}$ ) and drying under vacuum at $343 \mathrm{~K}$ for $4 \mathrm{~h}$. The catalyst weight, catalytic activity, and amount of product were measured after each reaction.

\section{Results and discussion}

\section{Catalyst characterization}

Fig. 1A shows the XRD patterns of MSN, Fe-MSN, and H/FeMSN. All samples exhibited ordered mesoporous silica and iron-containing structures with three diffraction peaks at $2 \theta=$ $2.26^{\circ}, 4.0^{\circ}$, and $4.54^{\circ}$, indexed as (100), (110), and (200) reflections, respectively. ${ }^{19}$ These peaks confirmed the presence of a two-dimensional hexagonal $(p 6 \mathrm{~mm})$ structure with a $d_{100^{-}}$ spacing of approximately $3.9 \mathrm{~nm}$. The presence of an intense peak at $2 \theta=2.26^{\circ}$ demonstrated a highly ordered mesoporous structure. Introduction of iron into the mesoporous silica nanoparticles (Fe-MSN) led to a reduction in the diffraction peaks due to the decreasing scatter contrast between the pore 

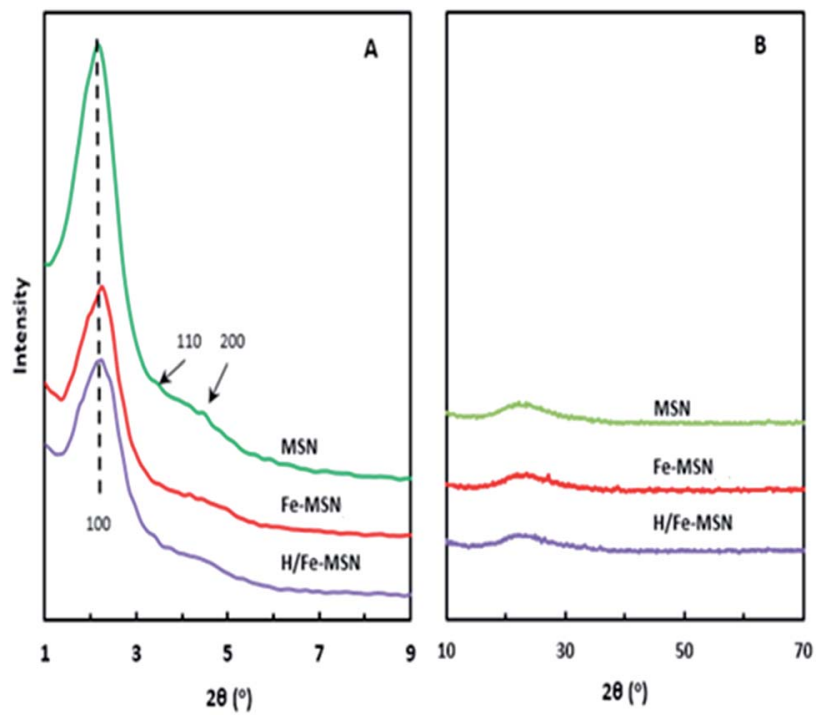

Fig. 1 XRD patterns of the MSN, Fe-MSN, and H/Fe-MSN catalysts. (A) Low region of $1-9^{\circ}$; (B) long region of $10-70^{\circ}$.

walls and space. ${ }^{20}$ Therefore, the order of crystallinity in Fe-MSN was lower. Protonation of Fe-grafted MSN (H/Fe-MSN) resulted in a decrease in the intensity of all peaks, similar to that of the Fe-MSN catalyst, which indicated that protonation occurred in the MSN framework. ${ }^{21}$ High-angle XRD patterns of MSN, FeMSN, and H/Fe-MSN are shown in Fig. 1B. No obvious diffraction peaks were observed in the high-angle diffraction range for incorporated iron in both Fe-MSN and H/Fe-MSN, suggesting the Fe species were well dispersed in the MSN framework.

Table 1 shows the physical properties of the MSN, Fe-MSN, and $\mathrm{H} / \mathrm{Fe}-\mathrm{MSN}$ catalysts. The surface areas of MSN, Fe-MSN, and $\mathrm{H} / \mathrm{Fe}-\mathrm{MSN}$ were 876,410 , and $858 \mathrm{~m}^{2} \mathrm{~g}^{-1}$, respectively. Lower surface areas were caused by changes in the pore size and/or the collapse of the 2D hexagonal structure of MSN. Introduction of iron into the MSN framework decreased the pore size from 3.15 to $2.60 \mathrm{~nm}$ and the pore volume from 0.35 to $0.10 \mathrm{~cm}^{3} \mathrm{~g}^{-1}$, which indicated the plugging of MSN pores with iron species. Protonation of Fe-MSN partially removed iron atoms inside the pores or walls, which reopened the pores of the structure. This was confirmed by the total pore volume, size, and wall thickness of these catalysts, which were analyzed using the BJH method (Table 1). Plug removal from Fe-MSN increased the total pore volume from 0.20 to $0.67 \mathrm{~cm}^{3} \mathrm{~g}^{-1}$ and the pore size from 2.60 to $3.53 \mathrm{~nm}$. Iron loading decreased the pore-wall thickness from 1.08 to $0.94 \mathrm{~nm}$ for MSN and Fe-MSN, respectively, which increased again to $1.14 \mathrm{~nm}$ in H/Fe-MSN due to widening of the pore size of MSN. Plug removal by protonation restored the pore size, volume, and wall thickness to almost original values.

XRF analysis showed Si/Fe molar ratios of 19.2 and 19.7 with iron amounts of 0.398 and $0.404 \mathrm{mmol} \mathrm{g}^{-1}$ in Fe-MSN and H/FeMSN, respectively (Table 2). Furthermore, the amount of iron in these samples was identified using ICP-OES (Varian ICP-OES 735), which confirmed the XRF results (Appendix A). The amount of $\mathrm{Fe}$ in these samples was close to the actual loading amount. Most of the loaded iron existed in the catalyst frameworks. Therefore, when protonating the iron-containing catalyst, the increase in the amount of Fe might be due to partial desilication of the Fe-MSN framework. ${ }^{22}$

Fig. 2A and B show nitrogen adsorption and desorption isotherms and the pore size distributions of MSN, Fe-MSN, and $\mathrm{Fe} / \mathrm{H}-\mathrm{MSN}$. All catalysts exhibited isotherms classified as Type IV with a Type $\mathrm{H} 4$ hysteresis loop, which can be attributed to their mesoporous structures (Fig. 2A).

The isotherms for these samples showed characteristic sharp inflections of capillary condensation within uniform pores at a $P / P_{0}$ of around $0.2-0.4$, which indicated the presence of small pore diameters and volumes. At a higher relative pressure $\left(P / P_{0}\right.$ of 0.8-1.0), no increase in adsorbed nitrogen was observed, which was attributed to Fe loading into the MSN framework and indicated to the success of the extra-framework structures. At a lower relative pressure $\left(P / P_{0}<0.2\right)$, a rounded transition confirmed the presence of a small number of micropores, whereas the pore size distribution confirmed the presence of a narrow peak at a pore diameter of around $1.21 \mathrm{~nm}$ (Fig. 2B). Iron grafting and protonation of Fe-MSN changed the MSN inflection isotherms at $<0.4$ and $>0.8$. The absence of hysteresis on the MSN confirmed that nitrogen sorption occurred on a nonporous surface material. These results verified that the MSN pores might be partially blocked by Fe atoms. Protonation of Fe-MSN recovered the MSN isotherm.

Table 2 Fe content in MSN, Fe-MSN, and H/Fe-MSN catalysts

\begin{tabular}{lll}
\hline Sample & Fe $\left(\mathrm{mmol} \mathrm{g}^{-1}\right)$ & XRF $(\mathrm{Si} / \mathrm{Fe})^{b}$ \\
\hline MSN & - & - \\
Fe-MSN $(\mathrm{Si} / \mathrm{Fe}=20)^{a}$ & 0.398 & 19.2 \\
$\mathrm{H} / \mathrm{Fe}-\mathrm{MSN}(\mathrm{Si} / \mathrm{Fe}=20)^{a}$ & 0.404 & 19.7
\end{tabular}

${ }^{a}$ Theoretical Si/Fe. ${ }^{b}$ Analyzed by XRF and ICP.

Table 1 Physical properties of MSN, Fe-MSN, and H/Fe-MSN catalysts ${ }^{a}$

\begin{tabular}{llllll}
\hline Sample & & & $S$ & & \\
$\left(\mathrm{~m}^{2} \mathrm{~g}^{-1}\right)$ & $V_{\mathrm{p}}\left(\mathrm{cm}^{3} \mathrm{~g}^{-1}\right)$ & & $W(\mathrm{~nm})$ \\
MSN & $d_{100}(\mathrm{~nm})$ & $a_{0}(\mathrm{~nm})$ & 876 & 0.35 & 3.15 \\
Fe-MSN & 3.93 & 4.20 & 410 & 0.20 & 2.60 \\
Fe/H-MSN & 3.80 & 3.54 & 858 & 0.67 & 3.53
\end{tabular}

${ }^{a} d_{100}, d$-value 100 reflections; $a_{0}$, pore center distance (equal to $\left.d_{100} \times 2 / \sqrt{ } 3\right) ; S$, BET surface area $\left(\mathrm{m}^{2} \mathrm{~g}^{-1}\right.$ ) obtained from $\mathrm{N}_{2}$ adsorption; $V_{\mathrm{p}}$, total pore volume $\left(\mathrm{mL} \mathrm{g}^{-1}\right)$; $W$, pore size (nm) obtained from BJH method; $t$, pore-wall thickness (equal to $a_{0}-W$ ). 

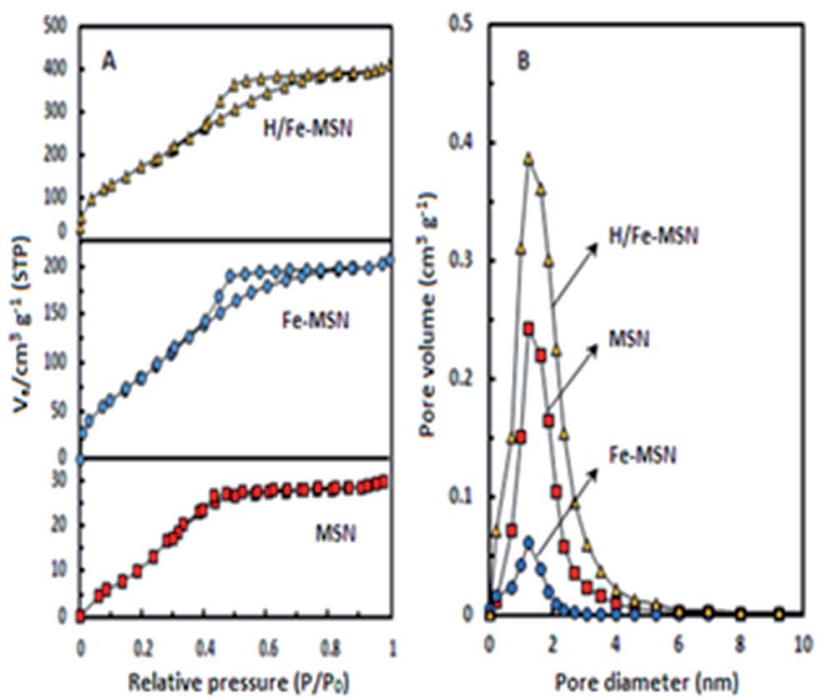

Fig. 2 (A) Nitrogen sorption isotherms and (B) pore size distributions for MSN, Fe-MSN, and H/Fe-MSN catalysts.

FTIR spectroscopy was used to determine structural differences between the MSN and iron-modified catalysts. Fig. 3A shows the FTIR spectra of the MSN samples in the range 3100$3400 \mathrm{~cm}^{-1}$. All MSN catalysts showed IR peaks attributed to SiO-Si bending (455 $\mathrm{cm}^{-1}$ ), Si-O-Si symmetric stretching (794 $\left.\mathrm{cm}^{-1}\right)$, Si-O-Si asymmetric stretching $\left(1050 \mathrm{~cm}^{-1}\right)$, and water molecules retained by silica materials $\left(1627 \mathrm{~cm}^{-1}\right) .^{23,24}$

FTIR spectra showed no major differences among the MSN, Fe-MSN, and H/Fe-MSN frameworks, which indicated that the MSN catalysts retained their siliceous structure even with iron incorporation. Notably, the bands relating to MSN at 455, 794, 1050 , and $1627 \mathrm{~cm}^{-1}$ were decreased by iron grafting, which might be due to the formation of ferrosilicate groups ( $\mathrm{Si}-\mathrm{O}-\mathrm{Fe}$ )
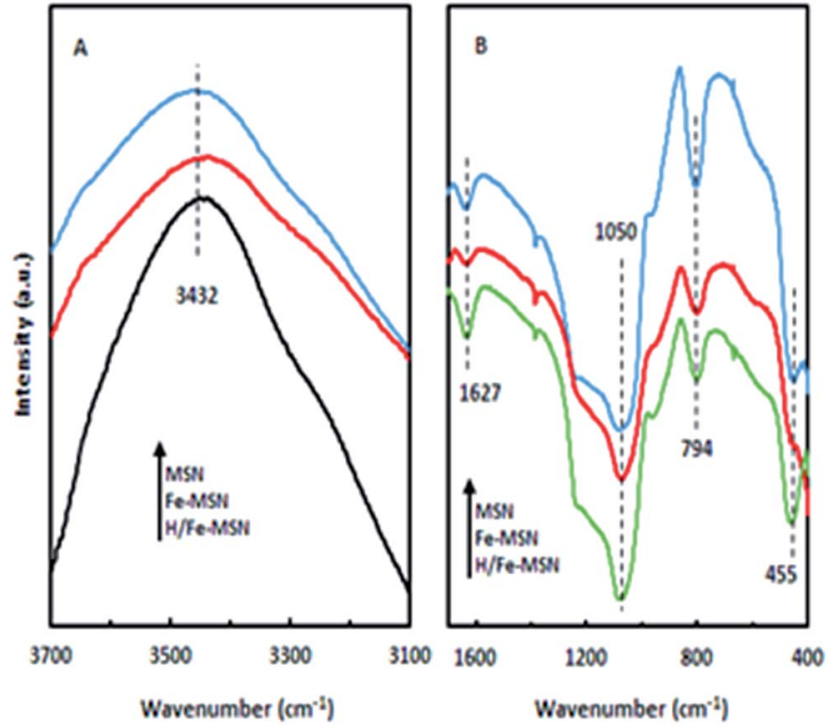

Fig. 3 FT-IR spectra of MSN, Fe-MSN, and H/Fe-MSN catalysts in the region (A) $3700-3100 \mathrm{~cm}^{-1}$ and (B) $1600-400 \mathrm{~cm}^{-1}$. in the framework. Fig. 3B shows the FT-IR spectra of these catalysts in the range $400-1600 \mathrm{~cm}^{-1}$. The vibration peak at $3432 \mathrm{~cm}^{-1}$ was attributed to hydroxyl groups in the catalyst structures. This peak was broad IR for pure MSN, but changed to sharper peaks in Fe-MSN and H/Fe-MSN, which indicated the decrease in hydrogen bonding in the Fe-containing samples. This change was due to the substitution of hydrogen in some hydroxyl groups with iron atoms.

The morphologies of MSN, Fe-MSN, and H/Fe-MSN were observed using FESEM and HRTEM images. The FESEM images (Fig. 4A-C) showed uniform spherical particles with particle sizes of 70-120 nm for MSN, Fe-MSN, and H/Fe-MSN. The TEM images (Fig. 4D-F) and their diffraction patterns (inset figures) clearly showed well-ordered pores with parallel and cylindrical channels and honeycomb structures, which indicated 2D hexagonal $p 6 \mathrm{~mm}$ mesostructures in these catalysts. ${ }^{3}$ These results were in accordance with the low-angle XRD patterns and nitrogen sorption analysis. XED (Fig. 5), ICP (Appendix A), and XRF (Appendix B) analyses confirmed the presence of iron in these nanomaterials.

Fig. 5 shows the EDX spectrum of the H/Fe-MSN catalyst. The presence of iron, silicon, oxygen, and carbon elements clearly confirmed the synthesis of H/Fe-MSN. According to this result, an iron loading of $2.63 \mathrm{wt} \%$ was present in $\mathrm{H} / \mathrm{Fe}-\mathrm{MSN}$, with ICP and $\mathrm{XRF}$ results $(\mathrm{Si} / \mathrm{Fe}=19.7)$ matching with the obtained results of EDX pattern analysis. Moreover, ICP and XRF analysis (Appendices A and B) and EDX results (not shown) indicated a molar ratio of $\mathrm{Si} / \mathrm{Fe}=19.2$ for $\mathrm{Fe}-\mathrm{MSN}$.

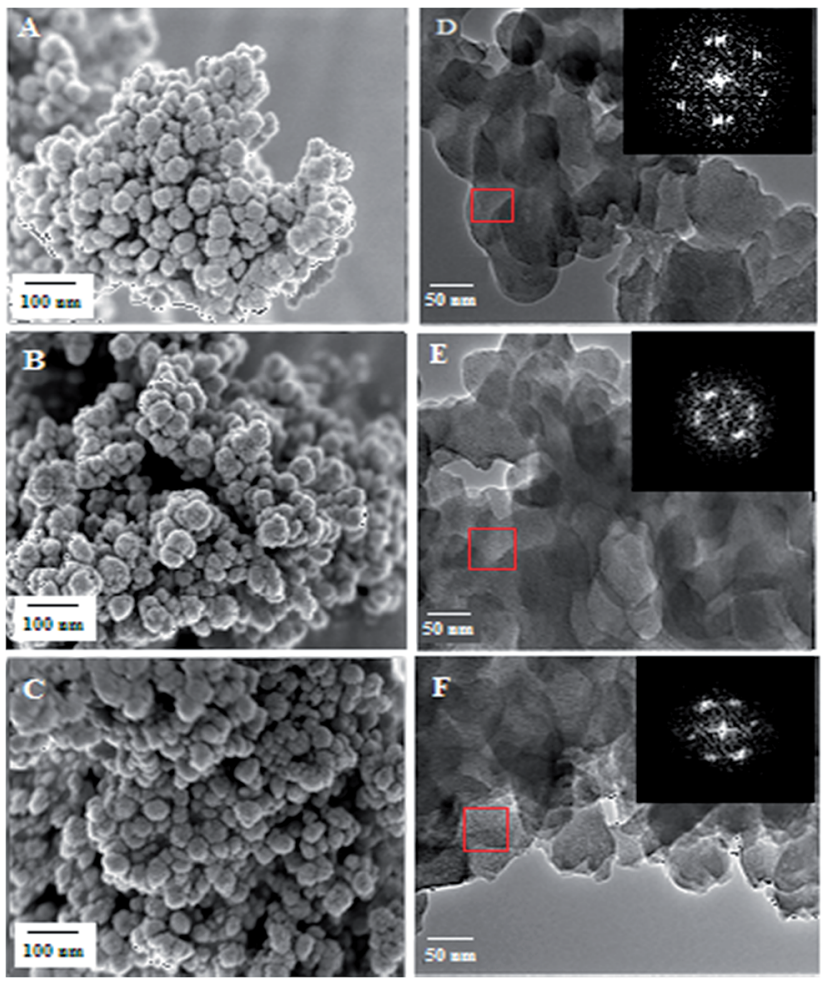

Fig. 4 FESEM images of (A) MSN, (B) Fe-MSN, and (C) H/Fe-MSN; and HRTEM images of (D) MSN, (E) Fe-MSN, and (F) H/Fe-MSN. 


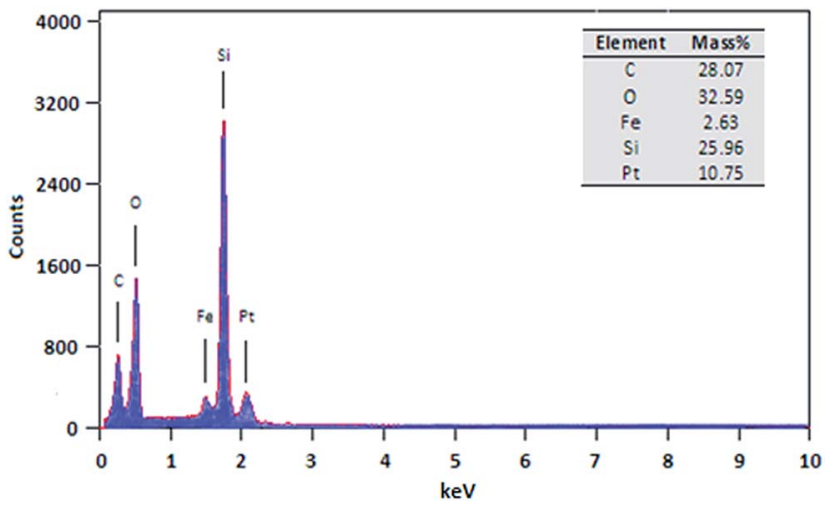

Fig. 5 EDX pattern of $\mathrm{H} / \mathrm{Fe}-\mathrm{MSN}$.

Fig. 6 shows the magnetic properties of $\mathrm{H} / \mathrm{Fe}^{\mathrm{np}}$-MSN catalyst through magnetic measurements of this catalyst at $298 \mathrm{~K}$ and 5 Tesla. The $\mathrm{Fe}^{\mathrm{np}}-\mathrm{MSN}$ and $\mathrm{H} / \mathrm{Fe}^{\mathrm{nP}}-\mathrm{MSN}$ samples exhibited magnetic moments of 3.3 and $4.1 \mathrm{emu} \mathrm{g}^{-1}$, respectively, and coercivities of about $13 \mathrm{kOe}$ at room temperature. Notably, the magnetic moment is calculated per total mass of the sample $\left(\mathrm{emu} \mathrm{g}^{-1}\right)$, including the total weight of iron oxide and silica. ${ }^{16}$ The prepared iron-coated samples displayed this magnetic property in their structures, which was increased in H/Fe-MSN, showing an enhanced amount of Fe in the framework, perhaps due to the desilication process. ${ }^{25}$

XPS spectra of the Fe-MSN and H/Fe-MSN catalysts are shown in Fig. 7. For both catalysts, two peaks were observed at $712 \mathrm{eV}$ and $725 \mathrm{eV}$, corresponding to the binding energies of Fe $2 \mathrm{p}_{3 / 2}$ and $\mathrm{Fe} 2 \mathrm{p}_{1 / 2}$. These two weak specific peaks showed variation satellites in the Fe 2p peaks. The H/Fe-MSN peak shifted by $1 \mathrm{eV}$ toward a higher binding energy, which indicated that the iron atoms were incorporated into the framework and that the $\mathrm{Fe}-\mathrm{O}-\mathrm{Si}$ bonds were stable. ${ }^{17,26}$

Cyclohexanone is an important compound used in the petrochemical industries and medicinal chemistry. Therefore, cyclohexanone can be considered a strategic material in different sciences. Selective oxidation of cyclohexane over some

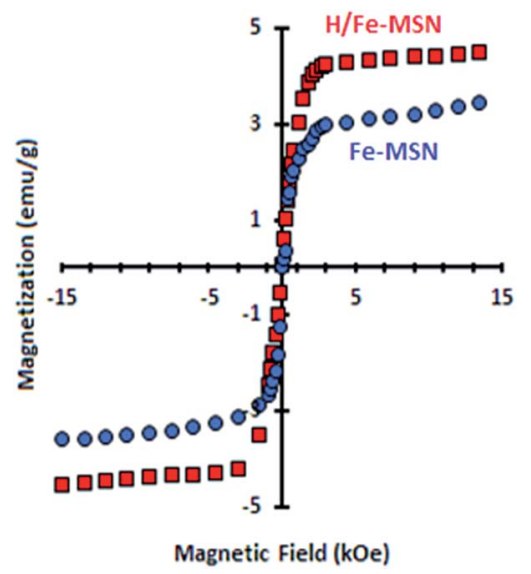

Fig. 6 Room temperature magnetization isotherms for $\mathrm{Fe}^{\mathrm{np}}-\mathrm{MSN}$ and $\mathrm{H} / \mathrm{Fe}^{\mathrm{np}}-\mathrm{MSN}$.

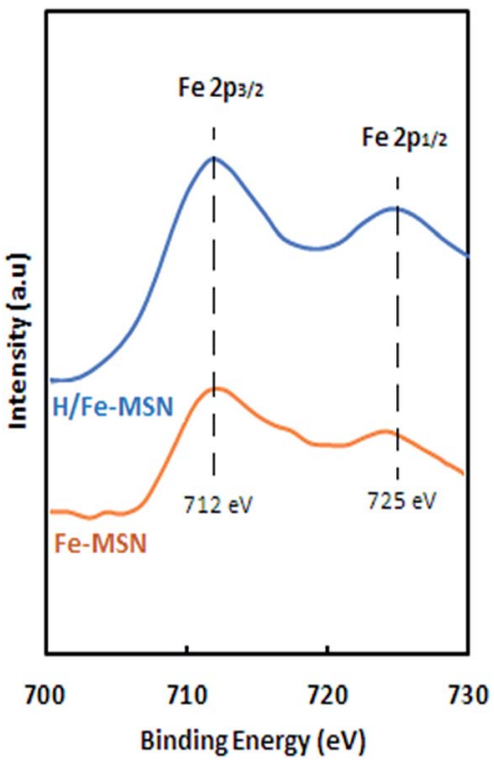

Fig. 7 XPS spectra of Fe-MSN and H/Fe-MSN.

nanomaterials under mild conditions has been of great interest to researchers and industry. ${ }^{27}$ Much effort has been made to determine mild conditions to promote oxidation conversion and the selectivity of cyclohexane using biomimetic systems to catalyze the selective oxidation of hydrocarbons at room temperature with different oxidants. Hydrogen peroxide is a suitable oxidant which can produce water and singlet oxygen during an environmentally friendly reaction. ${ }^{28}$ The results of classical cyclohexane oxidation with $\mathrm{H}_{2} \mathrm{O}_{2}$ shows low conversion and selectivity, ${ }^{29-32}$ which are major problems in the chemical industries.

Recently, Shul' pin et al. ${ }^{33}$ reported cyclohexane oxidation using a binuclear manganese(Iv) complex as catalyst in the presence of acetic acid to avoid $\mathrm{H}_{2} \mathrm{O}_{2}$ decomposition to water and oxygen. The reaction afforded cyclohexanone and cyclohexanol with $46 \%$ cyclohexane conversion at $293 \mathrm{~K}$ after $2 \mathrm{~h}$. A recent study catalyzed cyclohexene oxidation over metalloporphyrins with MCM-41 in the presence of aqueous $\mathrm{H}_{2} \mathrm{O}_{2}$. Metalloporphyrins containing $\mathrm{Fe}, \mathrm{Mn}$, and Co were encapsulated into the MCM-41 mesopore structure and hydrogen peroxide was used as a clean oxidant agent. ${ }^{18}$ The results showed that the supported metalloporphyrin complexes were the active sites for cyclohexene oxidation reactions, giving 26 , 12 , and $15 \%$ for $\mathrm{Fe}, \mathrm{Co}$, and Mn-immobilized metals, respectively.

This study concerns cyclohexane oxidation using pure MSN and iron-containing MSN catalysts under mild conditions. Fig. 8A shows cyclohexanone formation during cyclohexane oxidation over three catalysts: (a) pure MSN; (b) Fe-grafted MSN (Fe-MSN) with an initial $\mathrm{Si} / \mathrm{Fe}$ molar ratio of 20; and (c) protonated Fe-MSN (H/Fe-MSN). The results showed that the $\mathrm{H} /$ Fe-MSN catalyst exhibited higher performance in cyclohexane oxidation to form cyclohexanone than Fe-MSN and pure MSN. Both H/Fe-MSN and Fe-MSN showed the same drastically increasing trend in cyclohexanone formation. MSN has no 

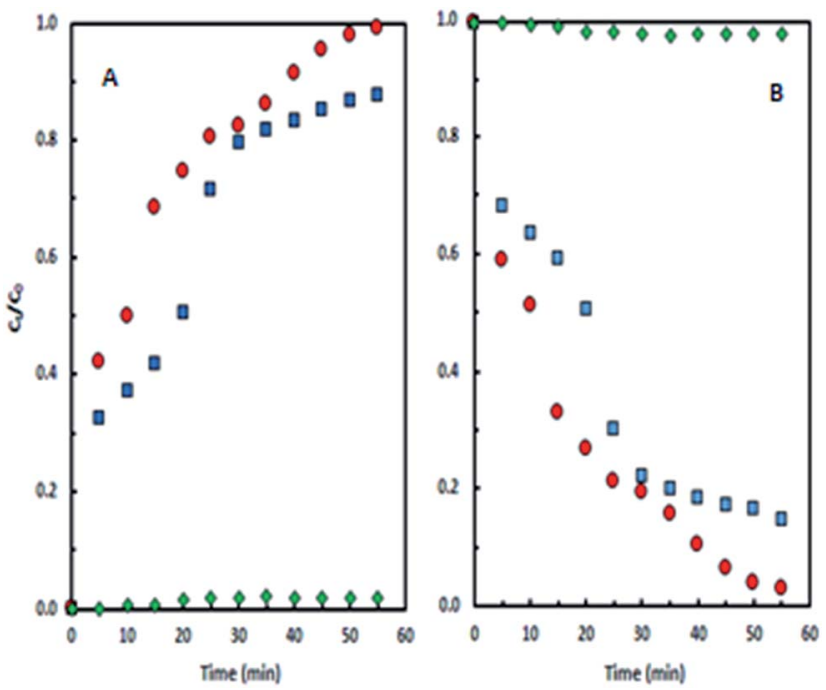

Fig. 8 (A) Cyclohexanone production with reaction time. (B) Cyclohexane consumption with reaction time.

catalytic activity due to the lack of acidic sites in its structure. ${ }^{2}$ The results indicate the high catalytic activity of $\mathrm{H} / \mathrm{Fe}-\mathrm{MSN}$, which formed about $0.8 \mathrm{~g} \mathrm{~L}^{-1}$ cyclohexanone after $25 \mathrm{~min}$, while Fe-MSN formed about $0.7 \mathrm{~g} \mathrm{~L}^{-1}$ in the same time.

Fig. 8B shows the consumption of cyclohexane during oxidation over MSN and Fe-modified MSN catalysts. MSN showed a constant amount of unreacted cyclohexane, while FeMSN and H/Fe-MSN showed a decreasing trend in cyclohexane consumption during the oxidation reaction. The cyclohexane consumption trend was almost converse to the cyclohexanone formation trend, which indicated the conversion of cyclohexane into cyclohexanone through oxidation.

\section{Proposed mechanism for cyclohexane oxidation reaction}

Published studies have described both free-radical and nonradical mechanisms that can be applied to the oxidation of olefins, aromatic hydrocarbons, and alcohols over metal oxides using $\mathrm{H}_{2} \mathrm{O}_{2} \cdot{ }^{34-36}$ Based on these studies, we propose a reasonable reaction mechanism for the oxidation of cyclohexane using hydrogen peroxide in the presence of $\mathrm{H} / \mathrm{Fe}-\mathrm{MSN}$ catalyst.

Scheme 1 shows the mechanism of cyclohexane oxidation to cyclohexanone over the H/Fe-MSN catalyst. Notably, the mechanism explained the active sites of tetrahedral $\mathrm{Fe}^{3+}$ on the catalyst surface. Hydrogen peroxide is activated on $\mathrm{H} / \mathrm{Fe}-\mathrm{MSN}$ by chemisorption on the iron surface, accompanied by the formation of a bi-radical Fe-peroxo complex. These radicals coordinate to Fe in $\mathrm{H} / \mathrm{Fe}-\mathrm{MSN}$ to form an iron-peroxo complex. This formed radical takes a hydrogen atom from a cyclohexane molecule to form a free biradical cyclohexanol form, followed by the attack of a carbon free radical to form cyclohexanone. ${ }^{37}$

The detailed product distribution of cyclohexane oxidation is listed in Table 3. At a low temperature ( $298 \mathrm{~K})$, cyclohexanone was observed as the main product using both catalysts. The $\mathrm{H} /$ Fe-MSN showed a higher cyclohexane conversion and cyclohexanone selectivity than Fe-MSN, which might be due to the

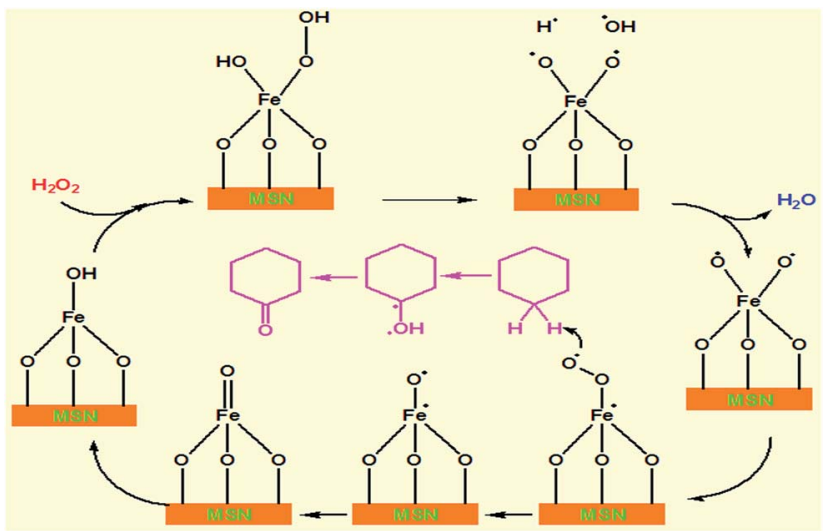

Scheme 1 Proposed mechanism of cyclohexanone synthesis from cyclohexane in the presence of $\mathrm{H}_{2} \mathrm{O}_{2}$ over $\mathrm{H} / \mathrm{Fe}-\mathrm{MSN}$ at $298 \mathrm{~K}$.

Table 3 Product distribution for cyclohexane oxidation over $\mathrm{H} / \mathrm{Fe}$ $\mathrm{MSN}$ at $298 \mathrm{~K}$ in $1 \mathrm{~h}$

\begin{tabular}{lll}
\hline & Fe-MSN & H/Fe-MSN \\
\hline Conversion (\%) & 86 & 97 \\
Selectivity (\%) & & \\
Cyclohexanone & 88 & 96 \\
Cyclohexanol & 12 & 4 \\
Yield (\%) & & \\
Cyclohexanone & 76.5 & 93.1 \\
Cyclohexanol & 9.5 & 3.9 \\
Cyclohexane & 14 & 3
\end{tabular}

presence of protonic sites. The selectivities for cyclohexanone formation were 88 and $94 \%$, and the cyclohexanone yields were 76.5 and $93.1 \%$, for Fe-MSN and H/Fe-MSN, respectively.

The ${ }^{1} \mathrm{H}$ and ${ }^{13} \mathrm{C}$ NMR spectra of the purified product of cyclohexane oxidation over H/Fe-MSN (Appendix C) confirmed the formation of cyclohexanone in high yield $\left({ }^{1} \mathrm{H}\right.$ NMR: d C4, $1.65 \mathrm{ppm}, 2 \mathrm{H}, \mathrm{t} ; \mathrm{C} 3,78 \mathrm{ppm}, 4 \mathrm{H}, \mathrm{m} ; \mathrm{C} 2,2.26 \mathrm{ppm}, 4 \mathrm{H}, \mathrm{m} ;{ }^{13} \mathrm{C}$ NMR: $\mathrm{CH}_{2}, 25$ ppm; $\mathrm{CH}_{2}, 27$ ppm; $\left.\mathrm{CH}_{2}, 42 \mathrm{ppm} ; \mathrm{CO}, 212 \mathrm{ppm}\right)$. Moreover, the measured boiling point $\left(154{ }^{\circ} \mathrm{C}\right)$ and melting point of the 2,4-dinitrophenylhydrazone derivative $\left(158-160^{\circ} \mathrm{C}\right)$ of the product confirmed that it was cyclohexanone formed in high yield.

The activities of Fe-MSN and H/Fe-MSN catalysts in the oxidation of cyclohexane was compared with recent studies using Fe-coated mesoporous/microporous silica nanomaterials as catalysts for the conversion of cyclohexane to cyclohexanone (Table 4). Although there are a few reports of cyclohexane oxidation over iron-containing mesoporous siliceous catalysts, none reported a high conversion of cyclohexane to cyclohexanone in a short time.

Fe-containing MCM-41 catalysts have exhibited 8.1 and $19.5 \%$ yields for cyclohexane oxidation. Furthermore, a very low number of active sites were observed in silica-supported iron oxide for the oxidation of cyclohexane. In this study, pure MSN showed a trace conversion of cyclohexane (2\%), while high 
Table 4 Comparison of activities of various Fe-containing silica-based catalysts for cyclohexane oxidation using $\mathrm{H}_{2} \mathrm{O}_{2}$ as oxidant

\begin{tabular}{|c|c|c|c|c|c|}
\hline Catalyst & Conv $^{a} \cdot(\%)$ & $m^{b}(\mathrm{wt} \%)$ & $T(\mathrm{~K})$ & $\begin{array}{l}t \\
\text { (h) }\end{array}$ & Reference \\
\hline MSN & 2 & 0.01 & 298 & 1 & This study \\
\hline $\mathrm{H} / \mathrm{Fe}-\mathrm{MSN}$ & 97 & 0.01 & 298 & 1 & This study \\
\hline Fe-MCM-41(1.64) & 8.1 & 0.1 & 373 & 12 & 38 \\
\hline Fe-MCM-41-NH & 19.5 & 0.1 & 373 & 12 & 39 \\
\hline
\end{tabular}

cyclohexane conversions of 86 and $97 \%$ were observed using the Fe-modified MSN catalysts in comparison with other mesostructured samples. The significant differences between Fegrafted MSNs and other samples are the high conversion of cyclohexane in a low temperature reaction within a short time.

All reactions in this study were carried out at room temperature $(298 \mathrm{~K})$ with a short time reaction of $1 \mathrm{~h}$, while the oxidation of cyclohexane required higher temperatures and long reaction times (more than $12 \mathrm{~h}$ ) over other catalysts. The high activity of $\mathrm{H} / \mathrm{Fe}-\mathrm{MSN}$ might be attributed to its high surface area, large pore size and volume, and the presence of $3 \mathrm{D}$ channels, which result in the high mass transfer of cyclohexane on the H/Fe-MSN surface. In the cyclohexane oxidation reaction, the turnover frequency (TOF) is the number of moles of cyclohexanone formed by a mole of Fe-MSN or H/Fe-MSN per unit time before becoming inactive. Here, the TOF was determined from the rate of cyclohexanone production relative to the amount of catalyst. The TOFs of cyclohexanone formed over FeMSN and H/Fe-MSN are shown in Fig. 9. Although, the TOF result exhibited an increasing trends for these catalysts with reaction time, the TOF using $\mathrm{H} / \mathrm{Fe}-\mathrm{MSN}$ was higher than that using Fe-MSN during a $1 \mathrm{~h}$ reaction.

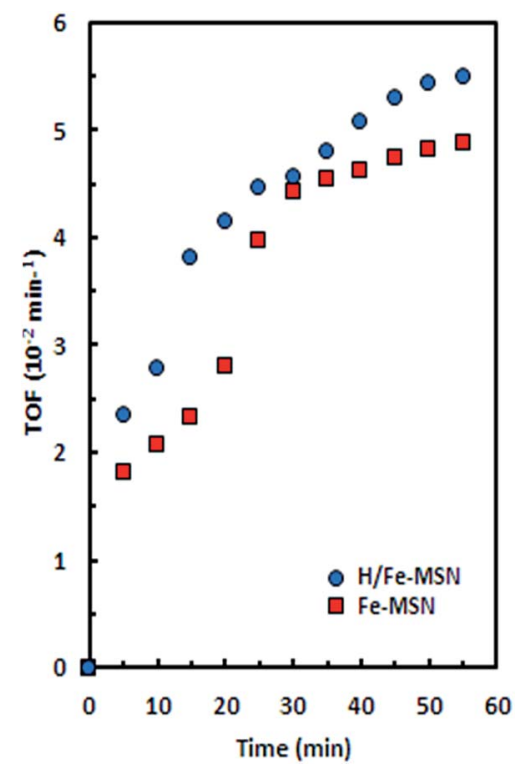

Fig. 9 Turnover frequency of cyclohexanone production during cyclohexane oxidation using $\mathrm{H}_{2} \mathrm{O}_{2}$ at $298 \mathrm{~K}$.

\section{Catalyst reusability}

The reusability of the H/Fe-MSN catalyst was investigated. After the oxidation reaction, the catalyst was recycled by washing with hot dichloromethane $(2 \times 10 \mathrm{~mL})$, drying under vacuum at 323 $\mathrm{K}$ for $3 \mathrm{~h}$, and then heating at $623 \mathrm{~K}$ under oxygen stream for $1 \mathrm{~h}$. Using this method, the catalyst was reused five times, and a minor loss in catalyst weight was observed after each recycle (total loss of 6\%). No significant decrease in the conversion of cyclohexane to cyclohexanone was observed. The yield
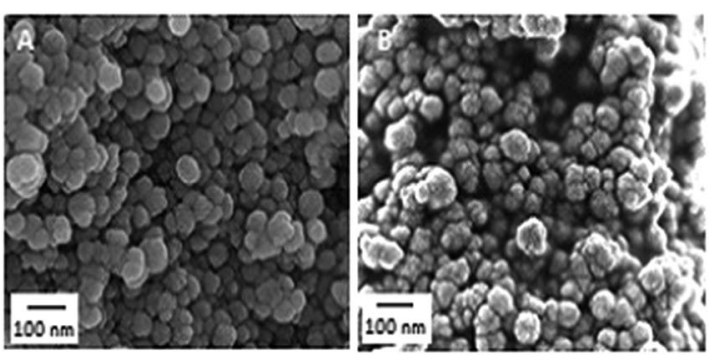

Fig. 10 FESEM images of (A) Fe-MSN and (B) H/Fe-MSN after cyclohexane oxidation reaction.
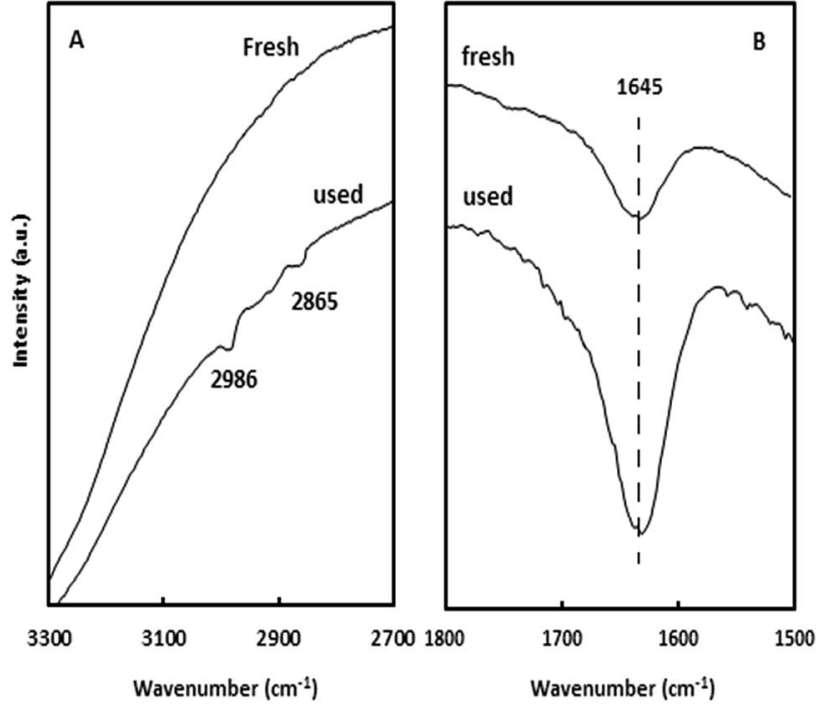

Fig. 11 FTIR spectra of H/Fe-MSN before (fresh) and after (used) reaction: (A) $\mathrm{C}-\mathrm{H}$ stretching and $(\mathrm{B}) \mathrm{C}=\mathrm{O}$ bond. 
decreased from 97 to $94 \%$, which exhibited the good reusability of this catalyst in the oxidation reaction.

Fig. 10 shows FESEM images of both Fe-MSN and H/Fe-MSN catalysts after the final oxidation reaction. The spherical particles indicated the stable structures of these nanomaterials. XRD patterns (not shown) indicated that there was no change after the oxidation reaction, but the presence of a carbon atom was observed in the used H/Fe-MSN, as shown in Fig. 11. Two weak bands observed at 2865 and $2986 \mathrm{~cm}^{-1}$ indicated the presence of a $\mathrm{C}-\mathrm{H}$ stretching vibration, which was related to the aliphatic group. The strong peak at $1645 \mathrm{~cm}^{-1}$, corresponding to the $\mathrm{C}=\mathrm{O}$ group, strongly confirmed the synthesis of cyclohexanone during this oxidation reaction.

\section{Conclusions}

Magnetic Fe-MSN and H/Fe-MSN solid acid catalysts were synthesized using a post-synthesis method from MSN followed by the protonation to afford H/Fe-MSN. X-ray diffraction patterns and nitrogen sorption results confirmed the mesoporous structures, with pore sizes of $3.9 \mathrm{~nm}$, and surface areas of 876,410 , and $858 \mathrm{~m}^{2} \mathrm{~g}^{-1}$ for MSN, Fe-MSN, and H/Fe-MSN, respectively. FTIR and XRD results confirmed that iron grafting followed by protonation carried out on the framework structure of MSN led to a decrease in the degree of catalyst crystallinity. XRF and ICP analysis showed $\mathrm{Si} / \mathrm{Fe}$ molar ratios of 19.2 and 19.7 for Fe-MSN and H/Fe-MSN, respectively. XPS spectra indicated the presence of $\mathrm{Fe}(\mathrm{II})$ and $\mathrm{Fe}(\mathrm{III})$ ions in the frameworks of these catalysts. Cyclohexanone, an important material in the petrochemicals industry, was produced over the Fe-MSN and H/Fe-MSN catalysts with high conversions of 86 and $97 \%$, and selectivities of 88 and $96 \%$, respectively, at $298 \mathrm{~K}$. The results showed that the conversion of cyclohexane using $\mathrm{H} /$ Fe-MSN was higher than that using Fe-MSN perhaps due to the presence of protonic sites. The advantages of the H/Fe-MSN solid catalyst include excellent cyclohexane conversion, low temperature, short reaction time, and fast work-up. This catalyst is also compatible with both organic and aqueous solvents.

The results of the oxidation reaction indicate that $\mathrm{H} / \mathrm{Fe}-\mathrm{MSN}$ can catalyze chemical reactions under mild, environmentally friendly conditions with excellent yield, short reaction time, and low energy consumption. The stable structure of the H/Fe-MSN catalyst allowed it to be recycled several times in oxidation reactions.

\section{Acknowledgements}

This work was assisted by the Research Council of North Tehran Branch, Islamic Azad University.

\section{References}

1 N. H. N. Kamarudin, A. A. Jalil, S. Triwahyono, V. Artika, N. F. M. Salleh, A. H. Karim, N. F. Jaafar, M. R. Sazegar, R. R. Mukti, B. H. Hameed and A. Johari, J. Colloid Interface Sci., 2014, 421, 6-13.
2 M. R. Sazegar, A. A. Jalil, S. Triwahyono, R. R. Mukti, M. Aziz, M. A. A. Aziz, N. H. N. Kamarudin and H. D. Setiabudi, Chem. Eng. J., 2014, 240, 352-361.

3 M. R. Sazegar, S. Mahmoudian, A. Mahmoudi, S. Triwahyono, A. A. Jalil, R. R. Mukti, N. H. N. Kamarudin and M. K. Ghoreishi, RSC Adv., 2016, 6, 11023-11031.

4 M. A. A. Aziz, A. A. Jalil, S. Triwahyono, R. R. Mukti, Y. H. Taufiq-Yap and M. R. Sazegar, Appl. Catal., B, 2014, 147, 359-368.

5 M. R. Sazegar, R. R. Mukti, S. Triwahyono, S. M. S. Mohaghegh, A. A. Jalil and M. Aziz, New J. Chem., 2015, 39, 8006-8016.

6 N. Thanabodeekij, W. Tanglumlert, E. Gulari and S. Wongkasemjit, Appl. Organomet. Chem., 2005, 19, 10471054.

7 Q. Zhang, Y. Wang, S. Itsuki, T. Shishido and K. Takehira, Chem. Lett., 2001, 946-947; CL-010566.

8 M. Popova, A. Szegedi, K. Lázár and A. Dimitrova, Catal. Lett., 2011, 141, 1288-1296.

9 H. R. Emamian, A. Honarbakhsh-raouf, A. Ataie and A. Yourdkhani, J. Alloys Compd., 2009, 480, 681-683.

10 Z. Surowiec, B. Bierska-Piech, M. Wiertel, M. Budzynski and J. Goworek, Acta Phys. Pol., A, 2008, 114, 1605-1612.

11 U. Schuchardt, D. Cardoso, R. Pereira, R. Sercheli, D. Mandelli, R. S. da Cruz, M. C. Guerreiro, E. V. Spinacé and E. L. Pires, Appl. Catal., A, 2001, 211, 1-17.

$12 \mathrm{~W}$. Thitsartarn, E. Gulari and S. Wongkasemjit, Appl. Organomet. Chem., 2008, 22, 97-103.

13 A. A. Costa, M. M. Santos, G. F. Ghesti, J. L. de Macedo, V. S. Braga, J. A. Dias and S. C. L. Dias, J. Mol. Catal. A: Chem., 2008, 282, 149-157.

14 M. R. Sazegar, S. Mahmoudian, A. Mahmoudi, S. Triwahyono, A. A. Jalil, R. R. Mukti, N. H. N. Kamarudin and M. K. Ghoreishi, RSC Adv., 2016, 6, 11023-11031.

15 N. H. N. Kamarudin, A. A. Jalil, S. Triwahyono, M. R. Sazegar, S. Hamdan, S. Baba and A. Ahmad, RSC Adv., 2015, 5, 3002330031 .

16 M. Arruebo, R. Fernández-Pacheco, B. Velasco, C. Marquina, J. Arbiol, S. Irusta, M. R. Ibarra and J. Santamaría, Adv. Funct. Mater., 2007, 17, 1473-1482.

17 A. De Stefanis, S. Kaciulis and L. Pandolfi, Microporous Mesoporous Mater., 2007, 99, 140-149.

18 A. A. Costa, M. M. Santos, G. F. Ghesti, J. L. de Macedo, V. S. Braga, J. A. Dias and S. C. L. Dias, J. Mol. Catal. A: Chem., 2008, 282, 149-157.

19 H. Wang, H. Tian and Z. Hao, J. Environ. Sci., 2012, 24(3), 536-540.

20 Y. L. Wei, Y. M. Wang, J. H. Zhu and Z. Y. Wu, Adv. Mater., 2003, 15(22), 1943-1945.

21 Y. M. Wang, Z. Y. Wu, Y. L. Wei and J. H. Zhu, Microporous Mesoporous Mater., 2005, 84(1-3), 127-136.

22 L. Wang, A. Wang, X. Li and F. Z. Y. Hu, J. Mater. Chem., 2010, 20, 2232-2239.

23 X. Du and J. He, J. Colloid Interface Sci., 2010, 345, 269-277.

24 S. M. Sidik, A. A. Jalil, S. Triwahyono, S. H. Adam, M. A. H. Satar and B. H. Hameed, Chem. Eng. J., 2012, 203, 9-18. 
25 B. Ellis and H. Foth, Soil Fertility, CRC press, 2nd edn, 1996, p. 46.

26 N. Wu, B. Li, J. Liu, S. Zuo and Y. Zhao, RSC Adv., 2016, 6, 13461-13468.

27 H. Nur, H. Hamid, S. Endud, H. Hamdan and Z. Ramli, Mater. Chem. Phys., 2006, 96, 337-342.

28 I. I. Moiseev, J. Mol. Catal. A: Chem., 1997, 127, 1.

29 S. Ito, T. Okuno, H. Matsushima, T. Tokii and Y. Nishida, J. Chem. Soc., Dalton Trans., 1996, 4479.

30 T. Okuno, S. Ohba and Y. Nishida, Polyhedron, 1997, 16, 3765-3774.

31 T. Okumo, S. Ito, S. Ohba and Y. Nishida, J. Chem. Soc., Dalton Trans., 1997, 3547-3551.

32 S. Nishino, H. Hosomi, S. Ohba, H. Matsushima, T. Tokii and Y. Nishida, J. Chem. Soc., Dalton Trans., 1999, 1509-1514.

33 G. B. Shul' Pin, G. Süss-Fink and J. R. L. Smith, Tetrahedron, 1999, 55, 5345-5358.
34 S. Dapurkar, A. Sakthivel and P. Selvam, J. Mol. Catal. A: Chem., 2004, 223, 241-250.

35 R. Neumann and M. Levin-Elad, Appl. Catal., A, 1995, 122, 85.

36 D. Huybrechts, P. L. Buskens and P. Jacobs, J. Mol. Catal. A: Chem., 1992, 71, 129.

37 L. Wang, S. Zhao, C. Liu, C. Li, X. Li, H. Li, Y. Wang, C. Ma, Z. Li and J. Zeng, Nano Lett., 2015, 15, 2875-2880.

38 W. A. Carvalho, P. B. Varaldo, M. Wallau and U. Schuchardt, Zeolites, 1997, 18, 408-416.

39 W. A. Carvalho, M. Wallau and U. Schuchardt, J. Mol. Catal. A: Chem., 1999, 144, 91-99.

40 R. S. da Cruz, M. M. Dauch, U. Schuchardt and R. Kumar, Stud. Surf. Sci. Catal., 2000, 130, 1037-1042.

41 N. Mizumo, I. Kiyoto, C. Nozaki and M. Misono, J. Catal., 1999, 181, 171-174. 